\title{
Pancreatic hemangioendothelioma, an extremely rare vascular anomaly in children: A case report and literature review
}

\author{
XIANG WANG and QIXING XIONG \\ Department of General Surgery, The Children's Hospital, Zhejiang University School of Medicine, \\ Hangzhou, Zheijiang 310003, P.R. China
}

Received September 28, 2014; Accepted May 12, 2015

DOI: 10.3892/ol.2015.3270

\begin{abstract}
The current study presents the case of a female infant with pancreatic hemangioendothelioma $(\mathrm{HE})$ and discusses this rare disease in terms of the clinical features, treatment principle and prognosis. An 8-month-old female with pancreatic HE was admitted to the Department of General Surgery in The Children's Hospital (Zhejiang University School of Medicine, Hangzhou, Zhejiang, China) on January 3, 2011, due to yellow skin and sclera that had been apparent for 1 week. Magnetic resonance imaging (MRI) showed dilatation in the left and right hepatic, common hepatic and common bile ducts, a pancreatic head mass of $\sim 4.7 \times 5.2 \mathrm{~cm}$ with a strong signal, and close linkage of the portal vein and mesenteric vessels to the mass. Laparotomy was performed on January 11, 2011. An intraoperative frozen specimen showed a benign tumor. Finally, Whipple surgery retaining the tumor was chosen. The patient was treated by fasting, gastrointestinal decompression, antibiotics and supportive treatment post-surgery. Jaundice disappeared rapidly following the surgery. The patient started eating at 1 week post-surgery and was discharged 1 week later. From the pathological results, a diagnosis of pancreatic HE was made, with no tumor invasion of the hepatic lymph nodes. During the follow-up visit at 28 months post-surgery, the child showed good growth and development. MRI showed that the mass size was $\sim 2.4 \times 2.0 \times 1.5 \mathrm{~cm}$, which was a significantly decrease from previously. Additionally, a literature search showed that from 1973 to the present date, only 9 studies on children with $\mathrm{HE}$ of the pancreas have been reported. Childhood pancreatic $\mathrm{HE}$ is a rare form of pancreatic vascular anomaly, mainly occurring in infants. If the tumor oppresses the duodenum and invades the mesenteric vessels, Whipple surgery retaining the tumor could be performed.
\end{abstract}

Correspondence to: Dr Xiang Wang, Department of General Surgery, The Children's Hospital, Zhejiang University School of Medicine, 57 Zhugan Lane, Hangzhou, Zhejiang 310003, P.R. China E-mail:wzy2081@163.com

Key words: pancreas, hemangioendothelioma, infant, prognosis

\section{Introduction}

Childhood pancreatic hemangioendothelioma (HE) is a rare form of vascular anomaly that mainly occurs in infants (1). Presenting signs and symptoms include obstructive jaundice, hepatomegaly, palpable mass, duodenal obstruction and intestinal bleeding (2). Ultrasound, computated tomography and magnetic resonance imaging scanning, not only help in localising the mass, but may also provide information on any associated findings (2). Differential diagnosis of pediatric pancreatic HE includes pseudopapillary tumor, pancreatoblastoma, rhabdomyosarcoma and endocrine cell carcinoma. The treatment of pancreatic HE is variable and dependent upon the clinical presentation such as biliary obstruction and vascular invasion (2). The present study reports a case of pancreatic HE in terms of its clinical features, treatment principles and prognosis through analysis of clinical symptoms, pre-operational examinations, intraoperational findings and pathological features. A literature review is also presented.

\section{Case report}

Patient information. An 8-month-old female was admitted to the Department of General Surgery in The Children's Hospital (Zhejiang University School of Medicine, Hangzhou, Zhejiang, China) on January 3, 2011, due to yellow skin and sclera that had been apparent for 1 week. The patient presented with acute onset of the disease, and the jaundice increased progressively. During the course of the disease, the child exhibited anorexia, yellow urine and white stool, with no abdominal pain, vomiting or fever. A physical examination upon admission to the hospital found listlessness, mild malnutrition, severe jaundice in the skin and sclera, and liver and spleen enlargement.

Laboratory outcome. Liver function tests showed a total bilirubin level of $270.3 \mu \mathrm{mol} / 1$ (1.71-17.1 $\mu \mathrm{mol} / \mathrm{l})$ and a direct bilirubin level of $248.6 \mu \mathrm{mol} / 1(0-3.4 \mu \mathrm{mol} / \mathrm{l})$, suggesting obstructive jaundice. Aspartate aminotransferase (254 U/1), alanine aminotransferase $(242 \mathrm{U} / \mathrm{l})$, pancreatic amylase and hepatitis-related tests all exhibited normal results.

Imaging characteristics. Ultrasonography suggested a pancreatic head body mass, distal common bile duct (CBD) obstruction and proximal CBD dilatation, with a diameter of 
$\sim 1.5 \mathrm{~cm}$. Magnetic resonance imaging (MRI) showed dilatation in the left and right hepatic ducts, the common hepatic duct and the CBD (Fig. 1), and a pancreatic head and body mass of $\sim 4.7 \times 5.2 \mathrm{~cm}$, with a strong signal. The portal vein and mesenteric vessels were closely linked to the tumor (Fig. 2). Chest computed tomography (CT) did not show any abnormalities.

Surgical procedure and treatment. Laparotomy was performed on January 11, 2011. During the surgery, the mass was found to be solid and tough, located in the head and body of the pancreas, and oppressing the CBD and duodenum. The mass surrounded the portal vein, superior mesenteric artery and vein, midcolic artery and vein, and was closely associated with the inferior vena cava. Consequently, the mass could not be removed. Examination of the spleen and adjacent retroperitoneal lymph nodes did not show any abnormalities. An intraoperative tumor frozen biopsy was performed. Tumor tissues, and a small piece of liver tissue and hepatic lymph node were fixed with formalin for inspection. The frozen specimen showed small round cells, which were possibly benign.

Palliative surgery was decided upon. The gallbladder was removed, the common hepatic duct was transected, and distal closure was performed for the common hepatic duct. The proximal and distal jejunum, which were already transected, were stitched with an end-to-side anastomosis. Disarticulation of the gastric antrum, removal of the pylorus and closure of the proximal duodenum were performed. Following formation of the antrum, the antrum and liver branch jejunum were stitched with an end-to-side anastomosis. The transected proximal jejunum and the liver branch jejunum $40 \mathrm{~cm}$ from the cholangio-intestinal anastomosis were stitched with an end-to-side anastomosis.

Due to the benign nature of this tumor, chemotherapy was not prescribed. The patient was treated by fasting, gastrointestinal decompression, administration of antibiotics (piperacillin sodium $100 \mathrm{mg} /$ day for 7 days) and supportive treatment (total parenteral nutrition, $75 \mathrm{Kcal} / \mathrm{day}$ for 7 days) following the surgery.

Clinical presentation. The post-operative jaundice disappeared rapidly. The patient started eating 1 week after the surgery and was discharged 1 week later. Liver function, pancreatic amylase level and other measures were all normal prior to discharge. Ultrasonography showed that the pancreatic mass had not increased in size, while a small amount of intraperitoneal effusion was observed.

Pathological results. Vascular endothelial cell proliferation was observed. A number of the cells formed a capillary with a small cavity, and there was less cellular atypia and collagen fiber hyperplasia between the tumor cells (Fig. 3). The immunohistochemical analysis showed that the tumor was cluster of differentiation $34^{+}$, vimentin ${ }^{+}$, $\mathrm{S}^{-} 00^{-}$, glucose transporter $1^{-}$, neuron-specific enolase ${ }^{-}$, chromogranin $\mathrm{A}^{+}$and epithelial membrane antigen ${ }^{+}$(Fig. 4). The liver biopsy results showed cholestatic liver cell swelling, mild portal area dilatation, small bile duct hyperplasia, bile plug formation and infiltration of a few inflammatory cells. A hepatic lymph node structure existed, but no tumor invasion was found. The consequent diagnosis was of pancreatic HE, with cholestatic hepatitis.

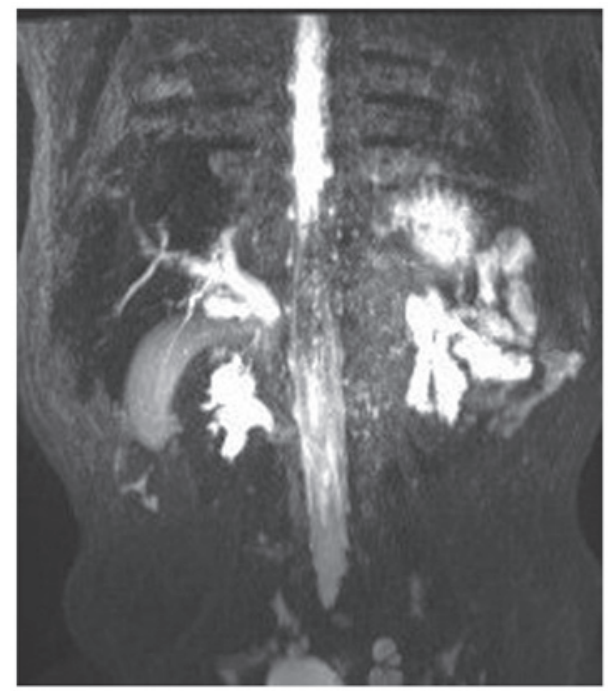

Figure 1. Magnetic resonance imaging scan showing the mass in the head of the pancreas compressing the common bile duct, leading to the dilatation of the intra- and extra-hepatic bile ducts.

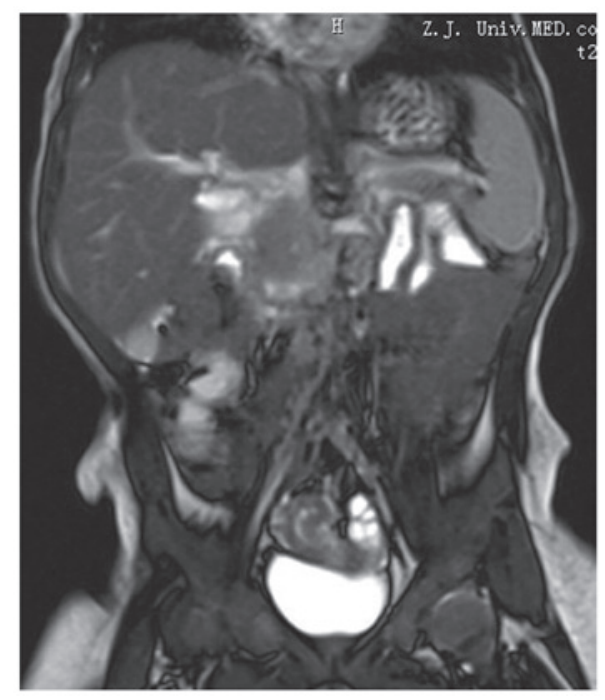

Figure 2. Pre-operative magnetic resonance imaging scan showing the tumor in the head of the pancreas encompassing the portal vein and mesenteric vessels, repressing the common bile duct and duodenum.

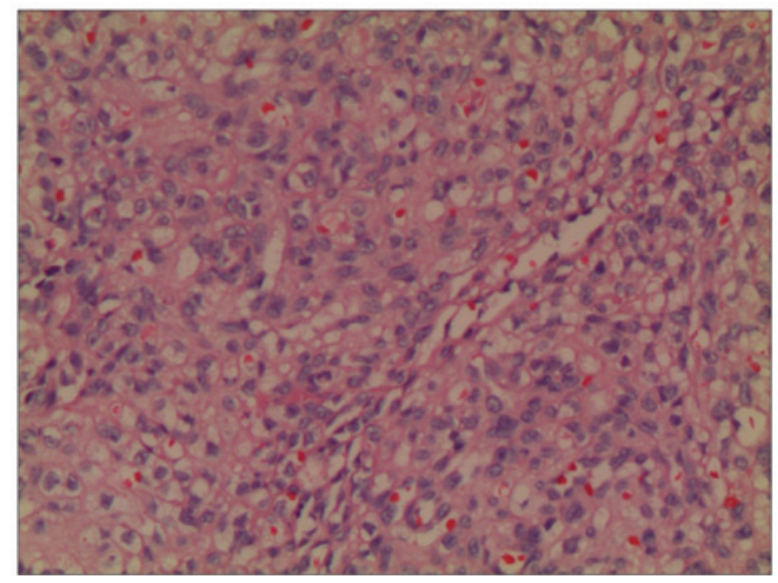

Figure 3. Pathological sections showing vascular endothelial cell proliferation, with less cellular atypia (hematoxylin and eosin stain; magnification, x20). 

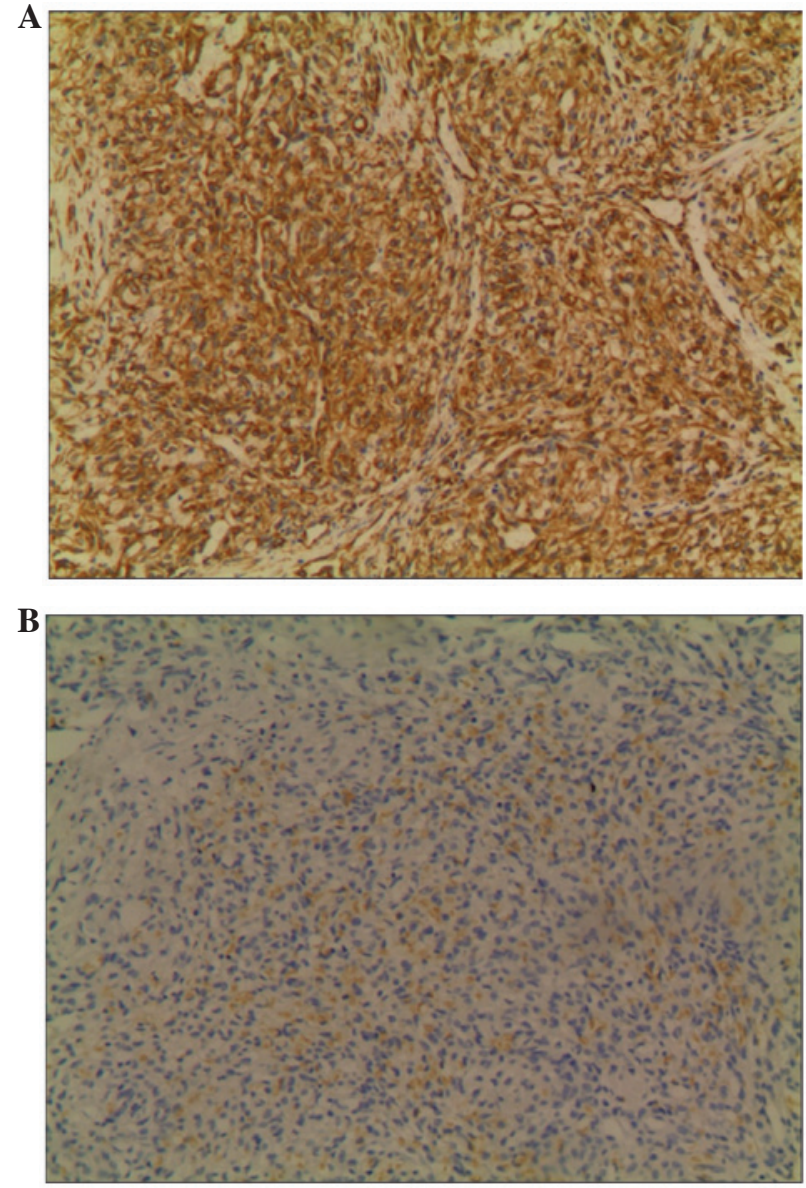

Figure 4. Immunohistochemistry showing (A) cluster of differentiation $34^{+}$ and (B) glucose transporter $1^{-}$tumor tissues.

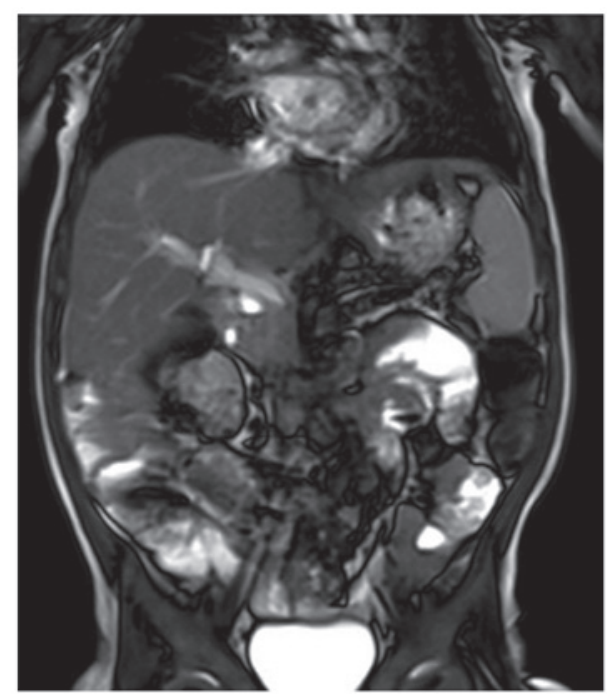

Figure 5. Magnetic resonance imaging scan showing a significantly reduced mass, with linkage to the mesenteric vessels and portal vein at 28 months post-surgery.

Follow-up. During the follow-up 3 months after the surgery, the patient showed an increase in body weight, no abdominal pain or jaundice, and urination and defecation were of a normal color. Liver function and pancreatic amylase levels were normal. Ultrasonography showed the size of the pancreatic

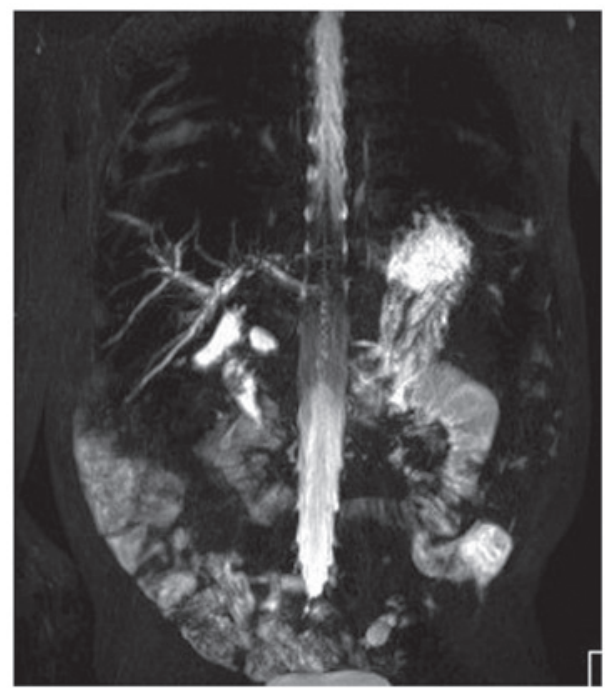

Figure 6. Magnetic resonance imaging showing mild intrahepatic bile duct dilatation and no pancreatic duct dilatation at 28 months post-surgery.

head mass to be $\sim 4.3 \times 3.6 \times 2.3 \mathrm{~cm}$, which was slightly reduced compared with the pre-operative size. During the follow-up at 28 months post-surgery, the child showed good growth and development, no abdominal pain, a good appetite, and normal urination and defecation. Upon examination, no jaundice was observed in the skin and sclera, and the abdomen was flat and soft. MRI showed the mass to be $\sim 2.4 \times 2.0 \times 1.5 \mathrm{~cm}$, which was a significantly reduced, but it was still closely linked to the mesenteric vessels and portal vein. No pancreatic duct dilatation and slight intrahepatic bile duct dilatation were observed, and there were no abnormal signals in the liver parenchyma (Figs. 5 and 6). Chest radiograph did not show any abnormalities.

\section{Discussion}

Pediatric congenital vascular abnormalities are extremely common, mostly occurring in the skin and soft tissues, with fewer observed in the organs, whether single or multiple in nature $(3,4)$. The liver is the most commonly involved organ (5). The most common histological type of this disease is HE, which rarely occurs in the pancreas (6).

PubMed was searched using the key words 'pancreas hemangioendothelioma infantile', 'pancreas kaposiform hemangioendothelioma', 'juvenile hemangioendothelioma' and 'spindle cell hemangioendothelioma'. The results showed that from 1973 to the present date, only 9 studies on children with HE of the pancreas have been published (7).

The case of a 3-year-old male with benign HE of the pancreas was reported by Horie et al in 1985 (8). Obstructive jaundice was the presenting symptom. The patient was treated with a temporary choledochojejunal bypass, with no additional treatment. In the 16-month follow-up period, the child remained well and asymptomatic. Laboratory data showed no abnormalities. A biopsy specimen of the pancreatic head showed a hemangiomatous pattern histologically. A positive reaction to factor VIII-related antigen and endothelial cell marker was noted on immunohistochemical study of the biopsy, supporting an endothelial origin. It was concluded that 
the prognosis of HE is favorable and that spontaneous regression can be expected, therefore, the initial tumor treatment should be conservative.

In 1987, Sauer et al reported the case of a 5-month-old female with jaundice and dilatation of the hepatic bile ducts (1). The pancreatic head and hepatic portal vein had a $3-\mathrm{cm}$ mass that completely blocked the gallbladder and CBD. Cholecystectomy surgery was performed, and a biopsy was performed of the tumor and regional lymph nodes. The pathological diagnosis was HE without lymph node invasion. Following the clear diagnosis, percutaneous biliary decompression was performed. Drainage continued for 22 months until the regression of the tumor. The study stated that this patient was the youngest case of percutaneous biliary drainage that has been reported.

In 1989, Villegas-Alvarez and Léon-Bojorge reported the case of a 6-month-old male with a pancreatic head vascular endothelial tumor, with Kasabach-Merritt syndrome (KMS) (9). The patient presented with thrombocytopenia and gastrointestinal bleeding. The authors believed that the vascular malformations and location of this disease gave this case great clinical significance. This disease commonly leads to neonatal obstructive jaundice and severe liver disease, eventually resulting in mortality. Paradoxically, the neoplasm is not considered malignant and the liver lesions that were produced secondarily could have been prevented if the patient was diagnosed correctly and at an earlier stage.

In 1993, Goldszmidt et al reported the case of a 2.5-month-old male admitted to hospital due to an abdominal mass (10). A routine blood test showed that the patient presented with severe anemia, with a hemoglobin level of $68 \mathrm{~g} / 1$ and a reduced blood platelet count of $50,000 / \mathrm{mm}^{3}$. Ultrasonography showed that the mass was retroperitoneal and heterogeneous. $\mathrm{X}$-rays delineated the mass, which distorted the duodenal loop and pressed forward against the stomach. Surgery showed that the mass included the pancreas, the root of mesentery and the extrahepatic bile duct. This mass was biopsied and histological examination showed infantile-type HE. Abdominal angiography showed that the mass was not particularly hypervascularized, and there were no dilated supplying blood vessels. The patient was administered methylprednisolone, but the volume of the mass remained unchanged, while hepatosplenomegaly and jaundice developed, and ultrasound showed dilated extra- and intrahepatic biliary ducts. The patient was then administered interferon- $\alpha 2 b$ for 1 month. Embolization of the small supplying arteries was performed due to a lack of improvement after 1 week of treatment. This procedure was followed by rapid disappearance of the signs of consumption coagulopathy, a progressive improvement in cholestasis and a decrease in the size of the mass (10).

In 2002, Lazure et al reported two cases of pancreatic vascular tumor, one of which was kaposiform $\mathrm{HE}$ and received tumor nourishing vascular embolization treatment with good effects (4). The study suggested that kaposiform HE has benign biological behaviors with a good prognosis.

Wang et al reported the case of a 9-month old infant with spindle cell HE of the pancreas in 2004 (10). Rapid intraoperative biopsy failed to confirm the diagnosis and the surgeons failed to remove the tumor, only employing interferon treatment after the pathological diagnosis. The tumor size was reduced, the jaundice disappeared and the follow-up visit showed good growth and development.

In 2006, Vogel et al collected 5,051 cases of children with vascular abnormalities (including vascular tumor and vascular malformations) who were treated at Boston Children's Hospital (Boston, MA, USA) between 1994 and 2004. Of these cases, 6 involved the pancreas and only 1 case was of kaposiform HE (2). The single case was in a male patient with tumor invasion of the porta hepatis, gallbladder, spleen, colon, omentum, mesentery and abdominal vascular structures, including the superior mesenteric artery, renal artery and celiac axis. The patient also presented with skin bruising and severe thrombocytopenia, fitting KMS. MRI, CT, angiography, colonoscopy and esophagogastroduodenoscopy (EGD) were used for the pre-operative examination and diagnosis of the patient. Next, systematic treatment was performed using corticosteroids and vincristine. It was suggested that palliative procedures, such as percutaneous drainage, stenting, and temporary or permanent bypass, should be performed for biliary obstruction and jaundice, and temporary or permanent bypass for intestinal obstruction. Pancreatectomy, in a variety of forms, can be used for the treatment of symptomatic lesions refractory to medical management and following the inability to exclude malignancy.

In a retrospective study, Park et al reviewed the clinical features and outcomes of all patients who were $<18$ years old with pancreatic neoplasms, and who were treated at Asan Medical Center (Seoul, South Korea) between December 1994 and March 2010 (11). A total of 32 patients were identified, but only 1 case of HE was found.

In the literature, only 9 patients have exhibited pancreatic $\mathrm{HE}$, with ages all but one under 1 year old, including 2 patients with KMS. The youngest was 2.5 months old, and the oldest was 3 years old. In 1940, Kasabach and Merritt reported the case of a newborn who exhibited rapidly increased hemangioma associated with thrombocytopenic purpura. Successive associated cases have been reported since that time. Researchers termed the systemic features of the syndrome with giant hemangioma associated with thrombocytopenia purpura as KMS (or hemangioma thrombocytopenia syndrome). KMS includes the severe consumption of platelets, a systemic bleeding tendency, an often acute onset and symptoms that worsen progressively during the short term. Generally, KMS occurs in children with giant hemangioma, while HE of the pancreas has a small volume; the reason for the associated KMS is unknown. The 2 infant patient cases recorded in the literature exhibited rapidly improved symptoms after the treatment, with hemorrhagic purpura disappearing and platelet counts returning to normal.

As the patient in the present study did not have symptoms such as skin vascular lesions or thrombocytopenia, it was difficult to diagnose the disease based only on pre-operative MRI and CT. Performing only percutaneous biliary drainage and drug treatment could lead to a risk of misdiagnosis. Therefore, although imaging results suggested that it would be hard to remove the tumor, surgical exploration was chosen for the sake of a clear diagnosis. As the tumor volume was large, severe oppression of the CBD and duodenum were observed, causing complete biliary obstruction and incomplete obstruction of the duodenum. Although the intraoperative frozen biopsy 
suggested a benign tumor, the tumor exhibited malignant biological behavior. As it surrounded the root of the mesenteric vessels and portal vein, and was hard to remove, only palliative surgery was considered. If only biliary drainage was performed, the problem of incomplete duodenal obstruction would not be solved. Thus, the final decision was made to perform Whipple surgery (biliary-enteric reconstruction) and retain the tumor. No post-operative medication, including hormones or interferon, was used. Good growth and development of the patient were observed for 6 months and the tumor exhibited a shrinking tendency. However, the long-term outcome remains to be observed. The prognosis may be good, but if the tumor grows gradually after surgery and leads to biliary or intestine obstruction again, certain medications such as corticosteroids or interferon may be prescribed..

In conclusion, childhood pancreas HE is extremely rare and is a benign vascular tumor that mostly occurs in infants. The tumor is likely to occur in the pancreatic head with oppression of the extrahepatic bile duct, leading to extrahepatic bile duct and duodenal obstruction. Formation of a clear diagnosis is difficult by pre-operative examinations, such as MRI, CT and angiography. Early surgical exploration and rapid frozen biopsy are recommended. If the intraoperative diagnosis of $\mathrm{HE}$ is confirmed, palliative surgery (biliary drainage) can relieve biliary obstruction. Medications such as corticosteroids and interferon are prescribed after the surgery; if the tumor is large with oppression of the duodenum and invasion of retroperitoneal vessels, Whipple surgery retaining the tumor can be performed.

\section{References}

1. Sauer L, Harrison MR, Bond SJ, Flake AW, Heyman MB and Ring EJ: Long-term percutaneous biliary drainage in an infant with hemangioendothelioma. J Pediatr Surg 22: 606-608, 1987.

2. Vogel AM,Alesbury JM,Fox VL and Fishman SJ: Complex pancreatic vascular anomalies in children. J Pediatr Surg 41: 473-478, 2006.

3. Mulliken JB, Fishman SJ and Burrows PE: Vascular anomalies. Curr Probl Surg 37: 517-584, 2000.

4. Lazure T, Tebboune N, Ben Lagha N, Triller MF, Pariente D and Fabre M: Pancreatic vascular tumours of childhood: A heterogeneous nosologic spectrum. Ann Pathol 22: 226-229, 2002 (In French)

5. Villegas-Alvarez $F$ and de Léon-Bojorge BY: Hemangioendothelioma of the pancreas and choledochus, as a cause of cholestatic neonatal and Kasabach-Merrit syndromes. Bol Med Hosp Infant Mex 46: 672-675, 1989 (In Spanish).

6. Villegas-Alvarez $F$ and de Léon-Bojorge BY: Hemangioendothelioma of the pancreas and choledochus, as a cause of cholestatic neonatal and Kasabach-Merrit syndromes. Bol Med Hosp Infant Mex 46: 672-675, 1989 (In Spanish).

7. Chappell JS: Case reports. Benign hemangioendothelioma of the head of the pancreas treated by pancreaticoduodenectomy. J Pediatr Surg 8: 431-432, 1973.

8. Horie H, Iwasaki I, Iida H, Takizawa J, Itoh $\mathrm{F}$ and Kohda S: Benign hemangioendothelioma of the pancreas with obstructive jaundice. Acta Pathol Jpn 35: 975-979, 1985.

9. Goldszmidt D, Pariente D, Yandza T, Dubousset AM and Valayer J: Kasabach-Merritt syndrome with pancreatic hemangioma in an infant. Arch Fr Pediatr 50: 593-597, 1993 (In French).

10. Wang HP, Ge L and Yu SY: The treatment of 5 cases with pancreas tumors in children. Academic journal of Shanghai Second Medical University 24: 691-692, 2004 (In Chinese).

11. Park M, Koh KN, Kim BE, Im HJ, Kim DY and Seo JJ: Pancreatic neoplasms in childhood and adolescence. J Pediatr Hematol Oncol 33: 295-300, 2011. 